\title{
Gas6 and the Tyro 3 receptor tyrosine kinase subfamily regulate the phagocytic function of Sertoli cells
}

\author{
Weipeng Xiong, Yongmei Chen, Huizhen Wang, Haikun Wang, Hui Wu, Qingxian Lu \\ and Daishu Han \\ Department of Cell Biology, School of Basic Medicine, Peking Union Medical College, Institute of Chinese Basic \\ Medical Sciences, Chinese Academy of Medical Sciences, 5 Dong Dan San Tiao, Beijing 100005, People's Republic of \\ China and ${ }^{1}$ Department of Ophthalmology and Visual Sciences, School of Medicine, University of Louisville, \\ Louisville, Kentucky 40202, USA
}

Correspondence should be addressed to D Han; Email: daishu@public.bta.net.cn

\begin{abstract}
The apoptotic spermatogenic cells and residual bodies are phagocytosed and degraded by Sertoli cells during spermatogenesis. The mechanisms of this process are largely unknown. Here, we demonstrate that Gas6 and its receptors, the Tyro 3 subfamily of receptor tyrosine kinases (RTKs; Tyro 3, Axl, and Mer), regulate the phagocytic function of Sertoli cells. The phagocytic ability of Sertoli cells increased by five times in the presence of Gas6 in serum-free medium when compared with controls. The Sertoli cells lacking Mer showed a $35 \%$ reduction in phagocytosis of apoptotic spermatogenic cells when compared with wild-type (WT) controls, whereas the Sertoli cells lacking Tyro 3 or Axl exhibited phagocytic activity comparable with the controls. Notably, the Sertoli cells lacking all three members of the Tyro 3 RTK subfamily showed a dramatic decrease in phagocytic ability of 7.6-fold when compared with WT Sertoli cells. The deficiency in phagocytosis by the triple-mutant Sertoli cells was due to the deficit in binding of the Sertoli cells to apoptotic germ cells. These findings suggest that Mer is responsible for triggering phagocytosis of apoptotic spermatogenic cells by Sertoli cells and that Tyro 3 , Axl, and Mer participate in recognizing and binding apoptotic germ cells by Sertoli cells in a redundant manner. Gas6 is a functional ligand of the Tyro 3 RTK subfamily in mediating phagocytic ability of Sertoli cells.
\end{abstract}

Reproduction (2008) 135 77-87

\section{Introduction}

The receptor tyrosine kinases (RTKs) are a superfamily of transmembrane proteins with a great diversity in their extracellular regions and a common highly conserved intracellular tyrosine kinase domain (Robinson et al. 2000). Intracellular signaling is triggered by activation of the tyrosine kinase domain and its subsequent phosphorylation of multiple substrates (Schlessinger 2000). Subfamilies of RTKs are categorized based on their amino acid sequence identities and extracellular structural similarities; thus, the members of a subfamily often bind common or similar ligands (Manning et al. 2002). The Tyro 3 RTK subfamily contains Tyro 3, Axl, and Mer (Hafizi \& Dahlback 2006b). Each of the three members is composed of two $\mathrm{N}$-terminal immunoglobulin-like domains and two fibronectin type III repeats in their extracellular regions. Two highly similar vitamin K-dependent proteins, Gas6 (product of growth arrestspecific gene 6) and protein $S$ (a negative regulator of blood coagulation), are the biological ligands of the Tyro 3 RTK subfamily (Hafizi \& Dahlback 2006a). These receptors and their ligands are widely expressed in cells of the immune, nervous, vascular, and reproductive systems. Genetic mutation studies have demonstrated that the three receptors play essential roles in these locations (Lu et al. 1999, Lu \& Lemke 2001, Lemke \& Lu 2003, Caraux et al. 2006).

More attention deserves to be paid to the functions of the Tyro 3 RTK subfamily on mammalian spermatogenesis. Mice triply mutant for the Tyro 3, Axl, and Mer genes completely lose the production of mature sperm (Lu et al. 1999). The impaired spermatogenesis in the triple-mutant mice is likely not due to germ cell autonomous defect, because all three members of the Tyro 3 RTK subfamily are expressed by Sertoli cells, but not by germ cells (Wang et al. 2005). Sertoli cells provide the tropic support to differentiating germ cells, and phagocytose the apoptotic germ cells and residual bodies during spermatogenesis.

As an intrinsic homeostasis, $70 \%$ of spermatogonia are estimated to undergo apoptosis under physiological conditions (Oakberg 1956, Huckins 1978, Johnson et al. 1983, Dym 1994). In later stages of spermiogenesis, the cytoplasmic portions of elongated spermatids are shed and form residual bodies before extrusion of 
differentiated sperm into the lumen of the seminiferous tubule (Kerr \& de Kretser 1974, Chemes 1986, Jegou 1991). Sertoli cells endocytose and degrade the residual bodies and apoptotic spermatogenic cells (Russell \& Clermont 1977, Chemes 1986, Pineau et al. 1991, Miething 1992), which is necessary for healthy spermatogenic cells to proceed through spermatogenesis (Ren \& Savill 1998). The mechanisms of this process remain to be clarified. It has been reported that Mer signaling is critical for the phagocytic clearance of apoptotic cells by professional phagocytes, such as macrophages (Scott et al. 2001, Cohen et al. 2002) and retinal pigment epithelium (RPE) cells (Hall et al. 2005, Prasad et al. 2006). We now demonstrate that Gas6 increases the phagocytic function of Sertoli cells in vitro, and Sertoli cells lacking the Tyro 3 RTK subfamily exhibit a compromised phagocytosis of apoptotic germ cells.

A conventional in vitro model to investigate the phagocytosis of apoptotic germ cells by Sertoli cells faced a problem in that it is complicated to discriminate the germ cells phagocytosed by Sertoli cells from those adhered to the Sertoli cells (Shiratsuchi et al. 1997, 1999). Early histological studies demonstrated that phagocytosis of apoptotic spermatogenic cells or residual bodies resulted in the formation of lipid droplets in Sertoli cells, which were last remnants of digested residual bodies or apoptotic cells (Chemes 1986). We recently established an in vitro approach to evaluate the phagocytosis of apoptotic spermatogenic cells by Sertoli cells through detecting lipid droplets using Oil Red O (ORO) staining (Wang et al. 2006). This method overcame the difficulty in distinguishing germ cells phagocytosed by Sertoli cells from those adhered to the
Sertoli cells. Here, using lipid droplets as a criterion, we demonstrate that the Tyro 3 subfamily receptors and their ligand, Gas6, regulate the phagocytosis of apoptotic germ cells by Sertoli cells.

\section{Results}

\section{Kinetics of lipid droplet formation in the primary cultured Sertoli cells}

Primary isolated Sertoli cells were always contaminated by spermatogenic cells, which led to the formation of abundant lipid droplets in the early days of culture of the primary Sertoli cells. The amount of lipid droplets decreased as the culture continued, particularly after removing the spermatogenic cells. In order to obtain the Sertoli cells with low levels of lipid droplets for phagocytosis assays, an analysis of the kinetics of lipid droplet formation during culture of the primary Sertoli cells was carried out. The ORO staining was performed every $24 \mathrm{~h}$ for 7 days after isolation, and the area ratios of lipid droplets to nuclei of Sertoli cells were determined using an image analyzer. The results are presented in Fig. 1. At $24 \mathrm{~h}$, abundant lipid droplets were detected (Fig. $1 \mathrm{~A}$ and $\mathrm{C}$ ), and the average area ratio of lipid droplets to nuclei was $17 \%$. This ratio decreased as the culture continued, and a sharp decrease appeared after removing spermatogenic cells at $48 \mathrm{~h}$ (Fig. 1C). At $96 \mathrm{~h}$, the lipid droplets reached a very low level (Fig. 1B and $\mathrm{C}$ ), and the area ratio of lipid droplets to nuclei was $3 \%$. No further evident decrease was observed after $96 \mathrm{~h}$. Therefore, the Sertoli cells at $96 \mathrm{~h}$ after isolation were used for phagocytosis assays.
A

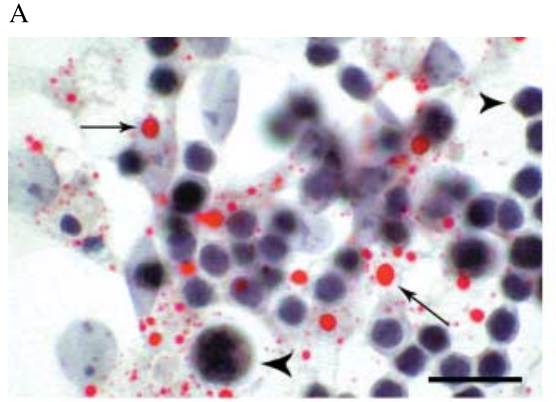

B

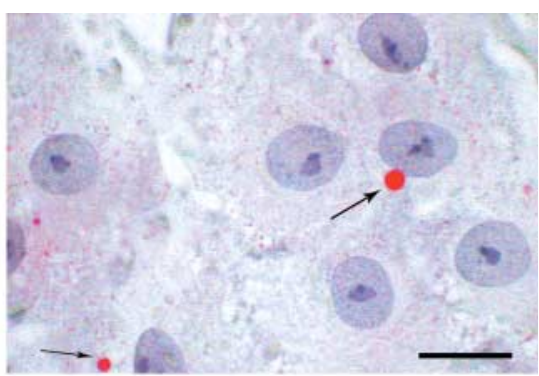

C

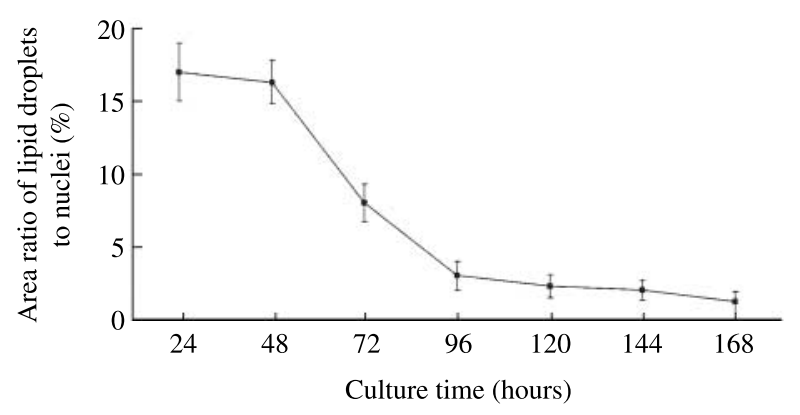

Figure 1 Dynamics of lipid droplet formation in primary Sertoli cells. The Sertoli cells were isolated from three mice ( 3 weeks old), and lipid droplets were detected by ORO staining at 24, 48, $72,96,120,144$, and $168 \mathrm{~h}$ after isolation. (A) Abundant lipid droplets (arrows) were observed in Sertoli cells at $24 \mathrm{~h}$, and different types of germ cells (arrowheads) were adhered on the Sertoli cells. (B) Only a few lipid droplets (arrows) were observed in Sertoli cells at $96 \mathrm{~h}$ after isolation. (C) Dynamics of lipid droplet formation in the Sertoli cells during culture. Area ratios of lipid droplets to nuclei of Sertoli cells were measured at each time point. The ratios decreased as the culture continued, and a sharply decreased ratio appeared after removing spermatogenic cells at $48 \mathrm{~h}$. The lipid droplets reached a very low level at $96 \mathrm{~h}$, and no more evident decrease was observed thereafter. Therefore, the Sertoli cells at $96 \mathrm{~h}$ after isolation were used for phagocytosis assays. Each datum was expressed as mean value (bar) \pm s.D. (error bar) of five repeat wells (40 cells/well). Scale bar $=20 \mu \mathrm{m}$. 


\section{Effects of Gas6 on the phagocytosis of apoptotic spermatogenic cells by Sertoli cells}

To assess whether Gas6 plays roles in regulating the phagocytic activity of Sertoli cells, a dose-dependent effect of Gas6 on the lipid droplet formation in Sertoli cells co-cultured with apoptotic germ cells in serum-free medium containing Gas6 were studied. At $24 \mathrm{~h}$ after co-culture in the presence of different concentrations of Gas6 $(0,10,30,50$, and $100 \mathrm{ng} / \mathrm{ml})$, the Sertoli cells were stained by ORO. A dramatic increase in lipid droplet formation appeared in the Sertoli cells cultured in serum-free medium containing $50 \mathrm{ng} / \mathrm{ml}$ Gas6 (Fig. 2A), and no further increase in the lipid droplet formation was observed in the presence of more Gas6 $(100 \mathrm{ng} / \mathrm{ml})$. Therefore, with $50 \mathrm{ng} / \mathrm{ml}$ Gas6 in the medium, a time-dependent effect of Gas6 on the lipid droplet formation was analyzed. At all three time points (Fig. 2B), the Gas6 significantly stimulated the lipid droplet formation in the Sertoli cells cultured in serumfree medium. Notably, a dramatic increase in lipid droplet formation was observed in the Sertoli cells cultured in medium containing serum when compared with that cultured in serum-free medium containing Gas6. However, fetal calf serum (FCS) and Gas6 had no additive effects on the lipid droplet formation in the Sertoli cells. These results suggested that Gas6 stimulated the phagocytic activity of Sertoli cells, and other factors in serum had effects on the phagocytosis of apoptotic spermatogenic cells. The pictures in Fig. 2C are representatives of images of lipid droplets in Sertoli cells at $24 \mathrm{~h}$ after co-culture with apoptotic germ cells, in serum-free medium with and without Gas6.

Binding assays showed that Gas6 significantly increased the binding of Sertoli cells to apoptotic germ cells about twofold (Fig. 2D) in serum-free medium. The increased binding by Gas 6 might contribute to the augmentation in phagocytosis of apoptotic cells by Sertoli cells cultured in medium containing Gas6.

To assess whether FCS and Gas6 affect expression of the Tyro 3 RTKs in cultured Sertoli cells, RT-PCRs were performed to determine mRNAs of Tyro 3 RTKs in Sertoli cells cultured with different conditions. At $24 \mathrm{~h}$ after culture, a similar mRNA level of Tyro 3 RTKs was detected in all culture conditions (Fig. 2E), suggesting that FCS and Gas6 did not change the expression of Tyro 3 RTKs.

\section{Phagocytosis of apoptotic spermatogenic cells by Sertoli cells mutant for Tyro 3 RTKS}

To address whether Gas6 acts on the phagocytosis by Sertoli cells through Tyro 3 RTKs, we analyzed the phagocytic ability of the Sertoli cells mutant singly, doubly, and triply for the Tyro 3, Axl, and Mer. First, we examined dynamics of the lipid droplet formation in the triple-mutant (Tyro $3^{-1-}, \mathrm{AxI}^{-/-}, \mathrm{Mer}^{-1-}$; TAM) Sertoli cells during primary culture in vitro. Compared with wild-type (WT) controls, TAM Sertoli cells showed a significantly lower level of lipid droplets until $96 \mathrm{~h}$ after isolation, and comparable lipid droplets appeared thereafter (Fig. 3A). It should be noted that the low level of lipid droplets in freshly isolated TAM Sertoli cells could not be affected by germ cells in vivo prior to isolation, because the same composition of germ cells existed in the seminiferous epithelium of WT and TAM mice at 3 weeks postnatal (data not shown). Second, phagocytosis assays were performed in WT and TAM Sertoli cells at $96 \mathrm{~h}$ after isolation. At $48 \mathrm{~h}$ after co-culture with apoptotic spermatogenic cells, the level of lipid droplets in WT Sertoli cells was 7.6-fold higher than that in TAM Sertoli cells (Fig. 3B). Figure 3C represents images of WT and TAM Sertoli cells stained by ORO after co-culture with apoptotic germ cells. These data suggest that the Tyro 3 RTKs mediate phagocytosis by Sertoli cells.

To determine the roles of different members of the Tyro 3 RTK subfamily in regulating phagocytosis by Sertoli cells, we compared the phagocytic activities of single, double, and triple Sertoli cell mutants. The results are shown in Fig. 3B. The Sertoli cells lacking single Mer exhibited a decrease in phagocytic ability by $35 \%$ compared with WT Sertoli cells. In contrast, the Sertoli cells lacking Tyro 3 or Axl did not show a difference in the level of lipid droplets when compared with the controls. To assess the possibility that different members of the Tyro 3 RTK subfamily act cooperatively in regulating the phagocytosis, the Sertoli cells doubly mutant for different combinations of the three receptors were analyzed for their phagocytic ability. The Sertoli cells lacking Tyro 3 and AxI (TA) exhibited comparable lipid droplet formation when compared with the controls. However, the level of lipid droplets in the Sertoli cells doubly mutant for Tyro 3 and Mer (TM) or for $\mathrm{Axl}$ and Mer (AM) decreased when compared with that in the controls, but comparable to that in the Sertoli cells singly mutant for Mer. Notably, the level of lipid droplets in the Sertoli cells lacking all three members of the Tyro 3 RTKs was 7.6-fold lower than that in WT controls and 4-fold lower than that in the single mutants for Mer. These results suggest that all three members of the Tyro 3 RTKs may be involved in regulating the phagocytic function of Sertoli cells; only Mer is necessary to initiate signaling to phagocytosis by Sertoli cells, while Tyro 3 and Axl did not cooperatively regulate the role of Mer.

\section{Binding of Sertoli cells to apoptotic germ cells}

To determine whether the deficiency in phagocytosis of apoptotic spermatogenic cells was due to the inability of mutant Sertoli cells to bind to apoptotic cells, the binding assays were performed. As shown in Fig. 4, the Sertoli cells from WT and Mer knockout mice bound to 
A

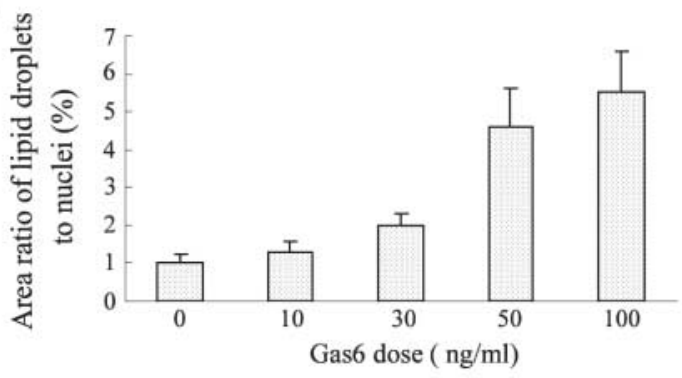

C

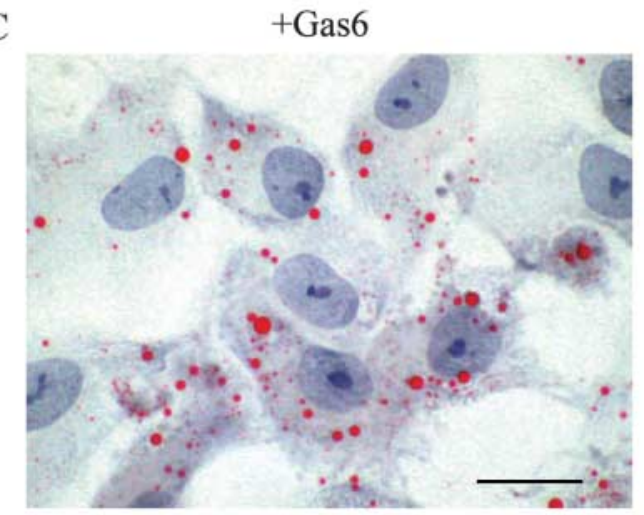

D

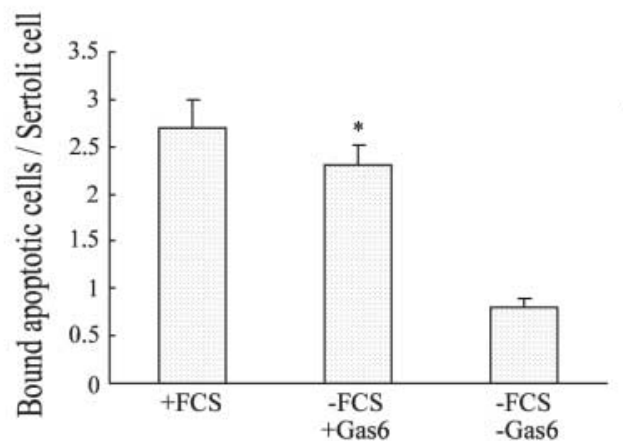

B

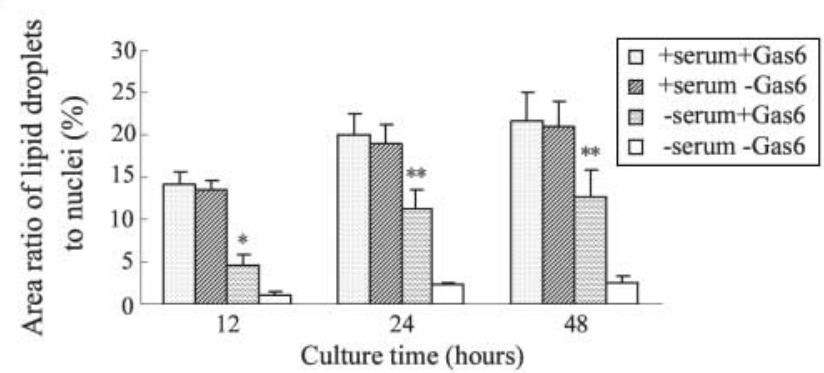

-Gas6

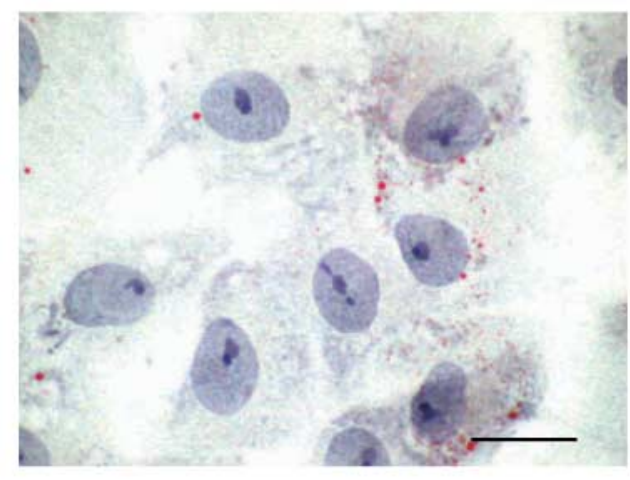

E

+ FCS

-FCS

-FCS

-Gas6

Tyro 3

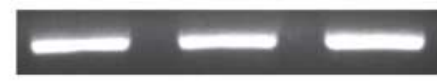

Axl

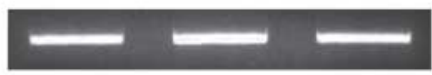

419 bp

Mer

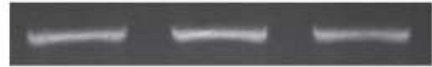

398 bp

325 bp

S16

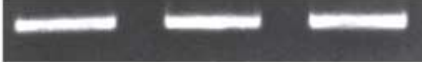

$326 \mathrm{bp}$

Figure 2 Effects of Gas6 on the phagocytic activity of Sertoli cells. Phagocytosis assays were performed by co-culture of Sertoli cells and apoptotic germ cells. In serum-free medium, Gas6 stimulated the phagocytic ability of Sertoli cells in a dose-dependent manner (A), and a plateau appeared at $50 \mathrm{ng} / \mathrm{ml}$ Gas6. With $50 \mathrm{ng} / \mathrm{ml}$ Gas6, a time-dependent phagocytosis by Sertoli cells was analyzed at different culture conditions (B). The formation of lipid droplets in Sertoli cells reached a plateau at $24 \mathrm{~h}$ after co-culture. Gas6 significantly increased the lipid droplet formation in the Sertoli cells co-cultured with apoptotic germ cells in serum-free medium. However, the level of lipid droplets in Sertoli cells cultured in serum-free medium containing Gas6 was significantly lower when compared with that in medium containing $10 \%$ FCS, indicating that more phagocytic inducers were present in serum. FCS and Gas6 did not show additive effects in culture. (C) Representatives of the images of lipid droplets in the Sertoli cells cultured in serum-free medium with or without Gas6 at $24 \mathrm{~h}$ after co-culture. Binding of apoptotic spermatogenic cells to Sertoli cells was examined at different culture conditions. Average bound spermatogenic cells per Sertoli cell represented binding efficiency of Sertoli cells (D). Gas6 significantly increased the binding of apoptotic germ cells to Sertoli cells. Semi-quantitative RT-PCRs were performed to analyze the expression of Tyro 3 RTKs in different culture conditions (E). At $24 \mathrm{~h}$ after culture, no difference was observed in the expression of Tyro 3 RTKs. The results suggest that FCS and Gas6 did not affect the expression of Tyro 3 RTKs. S16 rRNA was used as the control of equal amounts of cDNA in the PCRs. The pictures are representatives of three experiments. Each datum was expressed as mean value (bar) \pm s.D. (error bar) of five repeat wells $\left(40\right.$ cells $/$ well). ${ }^{* *} P<0.001$; ${ }^{*} P<0.05$. Scale bar $=20 \mu \mathrm{m}$.

apoptotic spermatogenic cells similarly with 2.8 and 2.6 per Sertoli cells respectively. All other single- and double-mutant Sertoli cells also showed similar binding ability to apoptotic spermatogenic cells. In contrast, the triple-mutant Sertoli cells showed a dramatic reduction in binding spermatogenic cells with an average of 0.8 per
Sertoli cell. These results suggest that Tyro 3 RTKs participate in the binding of Sertoli cells to apoptotic spermatogenic cells in a redundant manner. The significant reduction in binding apoptotic cells by the triple knockout Sertoli cells may contribute to the severe deficiency in phagocytosis. 

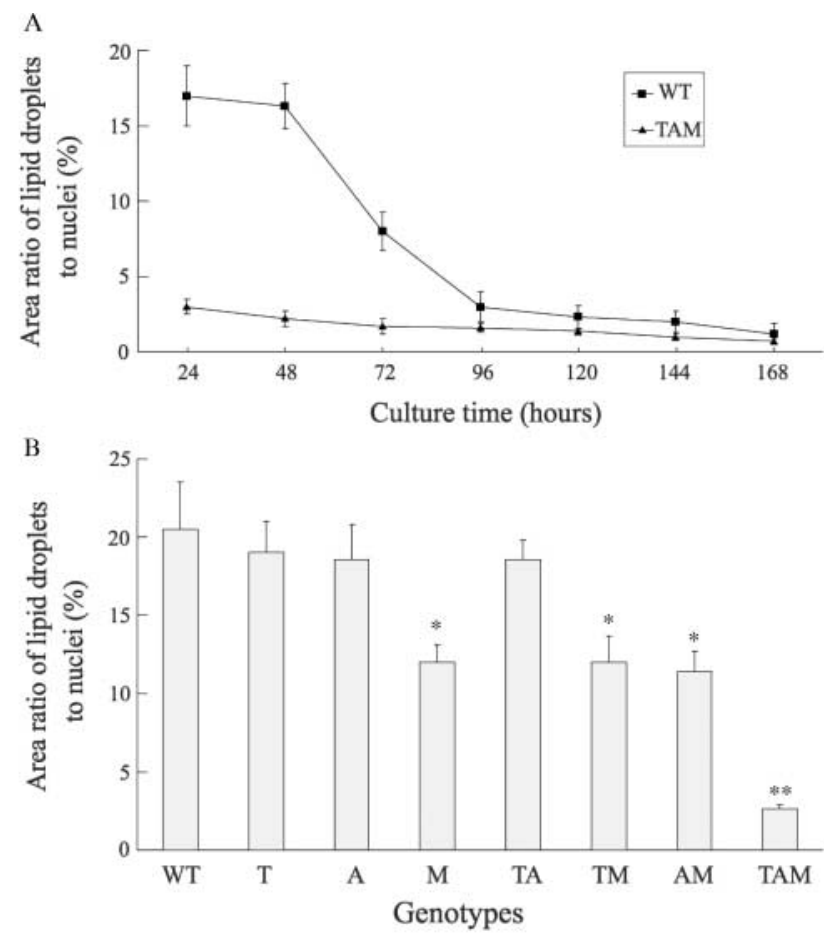

C WT
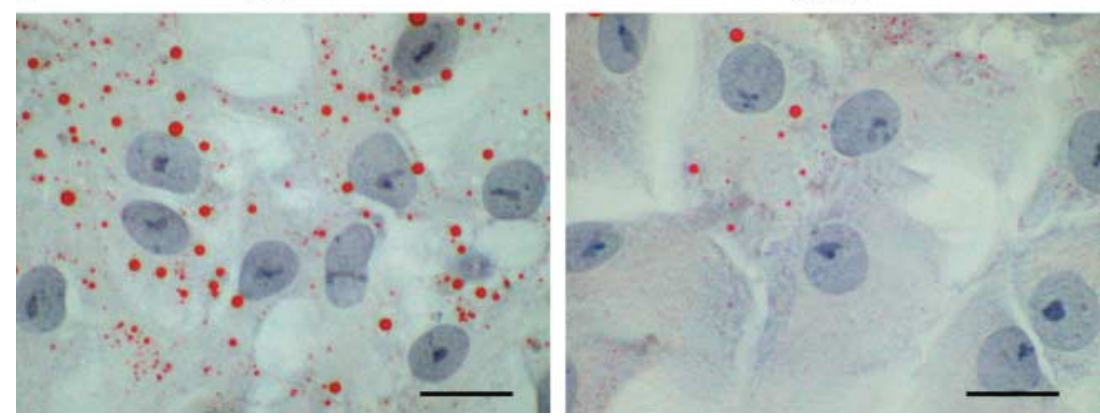

Figure 3 Analysis of the phagocytic ability of Sertoli cells mutant for Tyro 3 RTKs. (A) The primary Sertoli cells were isolated from three wild-type (WT) and triple-mutant (TAM) mice (3 weeks old) each, and lipid droplets were detected during culture with serum-containing medium. Significantly lower levels of lipid droplets were observed in TAM Sertoli cells at 24,48 , and $72 \mathrm{~h}$ after isolation when compared with that in WT Sertoli cells. (B) Phagocytosis assays were performed by coculture between Sertoli cells from each genotype and apoptotic spermatogenic cells in serumcontaining medium. Almost equal amounts of lipid droplets were observed in the Sertoli cells from WT, T (Tyro $\left.3^{-1-}\right), \mathrm{A}\left(\mathrm{AxI}^{-1-}\right)$, and TA (Tyro $3^{-1-}$ $\mathrm{Axl}^{-/-}$) mice. A significant decrease in lipid droplets was observed in the Sertoli cells from M $\left(\mathrm{Mer}^{-1-}\right), \mathrm{AM}\left(\mathrm{Axl}^{-1-} \mathrm{Mer}^{-1-}\right)$, and TM

(Tyro $3^{-1-} \mathrm{Mer}^{-1-}$ ) mice. A further decrease in lipid droplets formation was detected in the Sertoli cells from TAM mice. These observations suggest that Mer is required for the phagocytosis of apoptotic spermatogenic cells by Sertoli cells, and that Tyro 3 and Axl cooperatively enhance the role of Mer in regulating phagocytic ability of Sertoli cells. (C) Representative images of lipid droplets in WT and TAM Sertoli cells at $48 \mathrm{~h}$ after co-culture with apoptotic spermatogenic cells. Each datum was expressed as mean value (bar) \pm s.D. (error bar) of five repeat wells (40 cells/well). ${ }^{* *} P<0.001$; $* P<0.05$. Scale bar $=20 \mu \mathrm{m}$.

\section{Characterization of primary Sertoli cells}

To determine whether loss of Tyro 3 RTKs affects Sertoli cell viability and metabolism, which might influence the outcome of our study, we examined purity, survival rate, and metabolic activity of Sertoli cells during culture in vitro. Based on the immunostaining of WT 1 (Fig. 5A), the purity of Sertoli cells were more than $90 \%$ in all isolations. The survival rate of Sertoli cells was assessed by double staining with acridine orange and ethidium bromide (AO/EB). The nuclei of living cells gave a green fluorescence with $\mathrm{AO}$; the nuclei of dead cells gave an orange fluorescence with $\mathrm{EB}$; and the nuclei of apoptotic cells gave a yellow fluorescence with both $A O$ and $E B$ (Fig. 5B). Based on the staining, more than 95\% Sertoli cells survived within 2 weeks after isolation, and the ratio decreased slightly at 3 weeks after culture. More importantly, no difference on the survival rate was observed between WT and TAM Sertoli cells during culture (Fig. 5C). The general metabolic activity of Sertoli cells was assessed by methyl thiazolyl tetrazolium (MTT) tests. Until 3 weeks after isolation, WT and TAM Sertoli cells showed a comparable metabolic activity (Fig. 5D). Those observations indicated that loss of Tyro 3 RTKs did not affect viability and metabolic activity of the Sertoli cells in vitro, and the deficiency in phagocytosis of apoptotic cells could not be attributed to deficits in viability of TAM Sertoli cells during culture.

\section{Phagocytosis of latex beads by Sertoli cells}

To determine whether TAM Sertoli cells had a general defect in phagocytosis, we tested WT and TAM Sertoli cells for their ability to ingest latex beads. As shown in Fig. 6, there were no differences in the ingestion of latex beads between Sertoli cells from TAM and WT mice. The percentages of Sertoli cells that ingested latex beads were 25.1 and $24.5 \%$ for TAM and WT Sertoli cells respectively. By contrast, treatment with cytochalasin B 
A

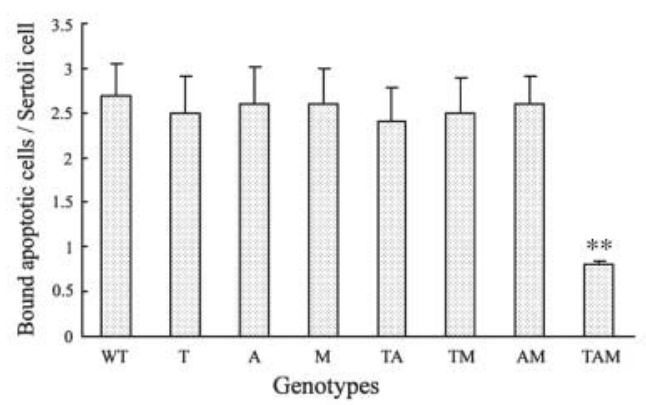

B

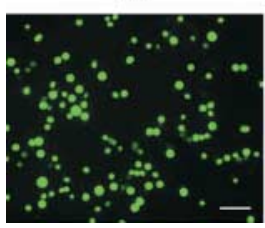

M

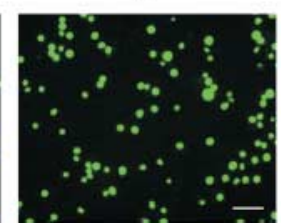

TAM

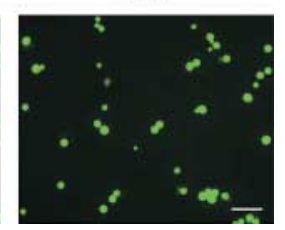

Figure 4 Binding of Sertoli cells to apoptotic spermatogenic cells. Sertoli cells with different genotypes were seeded in 24-well plates $\left(5 \times 10^{4}\right.$ cells/well). After $24 \mathrm{~h}$, fluorescein-labeled apoptotic spermatogenic cells were added to the Sertoli cells at $5 \times 10^{5} /$ well. After $1 \mathrm{~h}$ of incubation at $32{ }^{\circ} \mathrm{C}$, the co-cultures were washed five times with PBS. The spermatogenic cells bound to the Sertoli cells were scored under a u.v. microscope (IX-71, Olympus), and the Sertoli cells were counted under a light microscope. Average bound spermatogenic cells per Sertoli cell represented binding ability of Sertoli cells to spermatogenic cells (A). Assays were done in triplicate, counting a total of 150 Sertoli cells from three repeat wells each. (B) Representative images of bound spermatogenic cells to Sertoli cells from wild-type (WT), Mer knockout (M), and triple knockout (TAM) mice. ${ }^{* *} P<0.001$. Scale bar $=20 \mu \mathrm{m}$.

resulted in a marked decrease in the phagocytosis of latex beads by both WT and TAM Sertoli cells. These data show that the capacity of TAM Sertoli cells to engulf latex beads was maintained, and the phagocytic deficiency in TAM Sertoli cells was specifically restricted to the ingestion of apoptotic spermatogenic cells.

\section{Discussion}

The phagocytic clearance of apoptotic spermatogenic cells and residual bodies by Sertoli cells is necessary for spermatogenesis, and the mechanism of the phagocytosis by Sertoli cells remains to be clarified. In this study, we demonstrate that Gas6 and the Tyro 3 RTK subfamily participate in regulating phagocytic function of Sertoli cells.

Although most spermatogenic cells undergo apoptosis during spermatogenesis, only a limited number of apoptotic cells are detected histochemically. This is probably due to rapid elimination of apoptotic cells by phagocytosis. The fact that a few ingested particles were observed in Sertoli cells by ultrastructure studies on rat testis sections suggested that Sertoli cells degraded phagosomes rapidly (Chemes 1986). Therefore, the phagocytic ability of Sertoli cells could

be underestimated by examining directly the uptake of apoptotic cells. The lipid droplets in Sertoli cells were resulted from the phagocytosis of apoptotic spermatogenic cells, and could be a practical criterion to evaluate the phagocytic ability of Sertoli cells (Wang et al. 2006). In this study, the phagocytic activity of Sertoli cells was evaluated in vitro through detecting lipid droplets by ORO staining.

It has been reported that Gas6 mediates phagocytosis of dying cells by RPE cells (Hall et al. 2005) and macrophages (Scott et al. 2001). In this study, we demonstrate that Gas6 stimulates the phagocytosis of apoptotic spermatogenic cells by cultured Sertoli cells. Although Gas6 significantly increases the phagocytic ability of Sertoli cells in serum-free medium, it does not reach the level observed in the Sertoli cells cultured in medium containing 10\% FCS. Thus, Gas6 alone cannot substitute for $10 \%$ FCS. These observations suggest that other factors in FCS stimulate phagocytic activity of Sertoli cells. The fact that Gas6 and FCS do not have additive effects on the phagocytosis by cultured Sertoli cells suggests that excessive phagocytosis stimulators exist in FCS. In support of this expectation, the Gas6 mutant mice did not show any defect in the phagocytosis by phagocytes (Prasad et al. 2006). In fact, protein S, a closely Gas6-related molecule presented in serum, has been recently shown to stimulate the phagocytosis of apoptotic cells by RPE cells (Hall et al. 2005, Prasad et al. 2006), macrophages (Anderson et al. 2003), and neutrophils (Webb et al. 2003). Thus, we hypothesize that protein $\mathrm{S}$ could be a factor in serum to stimulate phagocytosis by Sertoli cells. Nevertheless, we cannot exclude the possibility that unknown phagocytosis inducers exist in serum.

Both Gas6 and protein S are common ligands for the Tyro 3 RTK subfamily (Hafizi \& Dahlback 2006a). It has been reported that phagocytic clearance of apoptotic cells by macrophages is mediated by Mer (Scott et al. 2001). A recent study demonstrated that both Mer and Tyro 3 acted on the regulation of phagocytosis by RPE cells (Prasad et al. 2006). Since all three members of the Tyro 3 RTK subfamily are expressed in Sertoli cells (Wang et al. 2005), the question of which member regulates the phagocytic activity of Sertoli cells is worthwhile to be addressed. To this end, we analyzed the phagocytosis of apoptotic spermatogenic cells by Sertoli cells mutant for Tyro 3 RTK subfamily receptors.

A previous study has implicated that Mer is a specific and sole RTK involved in the specialized phagocytosis of apoptotic cells in the immune system (Scott et al. 2001). It was demonstrated recently that Mer and Tyro 3, which were co-expressed by RPE cells, cooperatively participated in the regulation of phagocytosis by RPE cells (Prasad et al. 2006). We previously demonstrated that the proteins of all three members of Tyro 3 RTK subfamily were expressed by Sertoli cells (Wang et al. 2005). We now show that Mer is the sole member of the Tyro 3 RTKs for triggering 
A

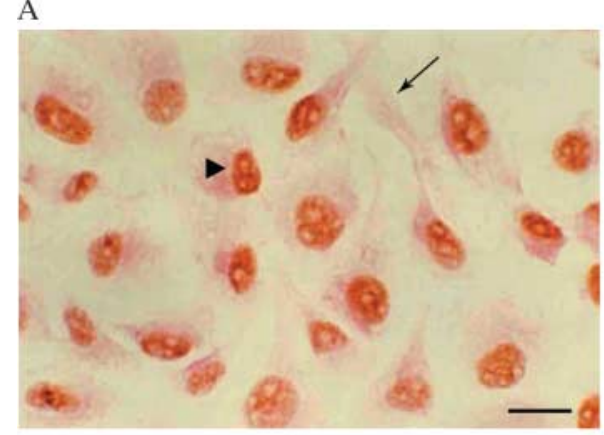

C

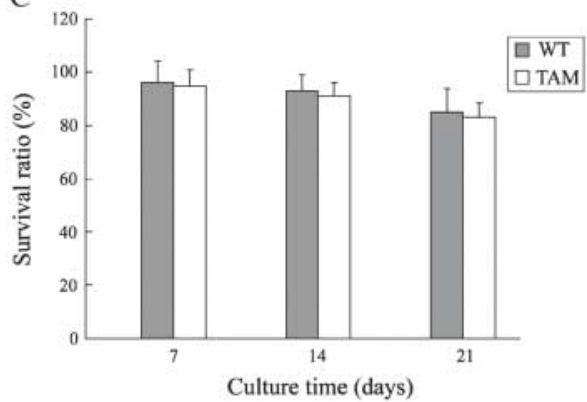

B

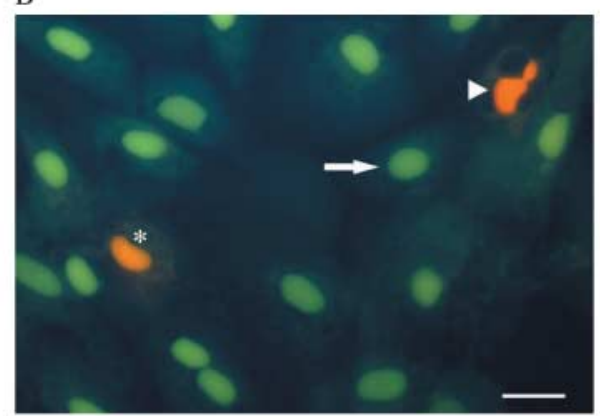

D

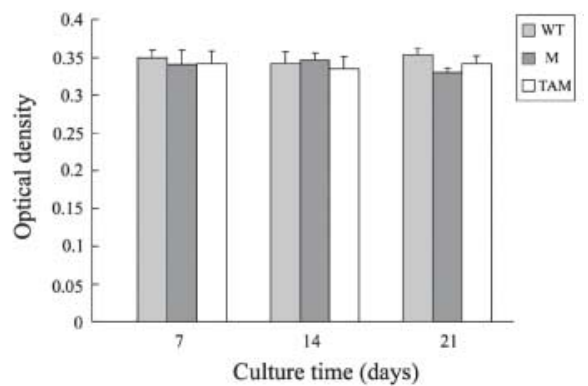

Figure 5 Characterizations of Sertoli cells. (A) The purity of Sertoli cells from different isolations was determined by immunostaining for WT 1. The nuclei of Sertoli cells were positively stained (arrowhead). The Sertoli cells were contaminated with a few cells stained negatively for WT 1 (arrow). Based on this analysis, the purity of Sertoli cells were more than $90 \%$ in all isolations. (B) The staining with dye mix of acridine orange and ethidium bromide (AO/EB) was used to assess Sertoli cells survival rate. The nuclei of living cells show a green fluorescence (arrow); the nuclei of dead cells show an orange fluorescence (arrowhead); and the nuclei of apoptotic cells give a yellow fluorescence (asterisk). Based on this method, more than 95\% cells survived within 2 weeks during primary culture, and no significant difference in the survival rate was observed between wild-type (WT) and triple-mutant (TAM) Sertoli cells (C). The metabolic activity of Sertoli cells was assessed by MTT test. Based on the reduction of yellow MTT to a purple formazan facilitated by dehydrogenases of metabolically active cells, no different metabolic activity appeared between WT and TAM Sertoli cells within 3 weeks culture (D). Each datum was expressed as mean value (bar) \pm s.D. (error bar) of three repeat assays, and three repeat wells were detected each assay. Scale bar $=20 \mu \mathrm{m}$.

phagocytosis by Sertoli cells. However, all three Tyro 3 RTKs participate in binding of Sertoli cells to apoptotic spermatogenic cells in an overlapping manner, which facilitates the phagocytic function of Sertoli cells. The cooperative functions of the Tyro 3 RTK subfamily have been reported with respect to regulation of natural killer (NK) cell differentiation and megakaryocytopoiesis (Caraux et al. 2006, Wang et al. 2007). Consistent with the functional cooperation, the members of the Tyro 3 RTK subfamily are frequently co-expressed within the same cell types, such as NK cells, and megakaryocytes. The co-expression of Tyro 3 RTK subfamily members may facilitate their functional cooperation.

The recognition of apoptotic cells by phagocytes involves engulfment receptors on the phagocytes. These receptors may simply tether the corpse to the phagocyte or may also initiate signaling pathways for engulfment. The results of binding assays showed that the existence of any one of the Tyro 3 RTKs in Sertoli cells exhibited similar binding to apoptotic germ cells, and loss of all three Tyro 3 RTKs resulted in a significant reduction in binding. These results suggest that all three members of the Tyro 3 RTKs might serve to tether apoptotic spermatogenic cells to Sertoli cells. The binding of the apoptotic cells to Sertoli cells mediated by Tyro 3 RTKs could not be homologous adhesion, since none of the Tyro 3 RTKs was expressed by spermatogenic cells (Wang et al. 2005). A previous study demonstrated that Gas6 mediated cell adhesion to phosphatidylserine (PS; Nakano et al. 1997). In this study, we demonstrated that Gas6 promoted binding of Sertoli cells to apoptotic spermatogenic cells. The binding could be mediated by Tyro 3 RTKs through an interaction with Gas6, which binds to PS on the surface of apoptotic cells. It has been reported that the ability of serum to stimulate phagocytosis in cultured human macrophages is due to the presence of protein $S$ in serum and the ability of protein $\mathrm{S}$ to bind to PS exposed on the surface of apoptotic cells (Anderson et al. 2003). The apoptotic rat spermatogenic cells were phagocytosed by Sertoli cells in a PS-mediated manner, in which class B of scavenger receptor type I functioned as a PS-recognizing phagocytosis receptor of Sertoli cells (Shiratsuchi et al. 1997, 1999, Nakagawa et al. 2005). We therefore speculate that Gas6 binds firstly through its Gla domains to the PS exposed on the surface of apoptotic spermatogenic cells and secondly through its steroid hormone-binding globulin domain to its receptors, which provides a bridge between Sertoli cells and apoptotic spermatogenic cells. Although a recent 

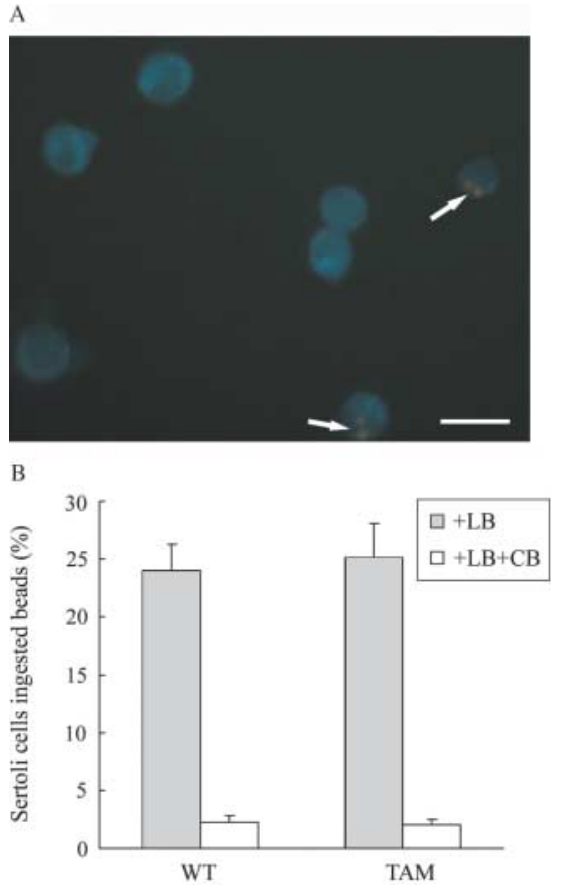

Figure 6 Uptake of latex beads by Sertoli cells. Sertoli cells were co-cultured with yellow fluorescence latex beads. At $6 \mathrm{~h}$ of co-culture, cells were washed five times vigorously and treated with $0.05 \%$ trypsin to remove the beads bound on the surface of the cells. The cells were counted under a fluorescence microscope (IX-71, Olympus) and the proportion of cells having internalized beads represented the results. (A) Image of Sertoli cells with engulfed latex beads (arrows). (B) The ratio of the Sertoli cells having internalized latex beads (LB). Inhibition of actin involvement by cytochalasin B (CB) in the phagocytic activity was performed simultaneously. A total of 200 cells were counted at each test, and the results were expressed as the mean value (bar) \pm s.D. (error bar) of five tests. Scale bar $=20 \mu \mathrm{m}$.

study showed that Mer played a role in alphavbeta 5 integrin-mediated phagocytosis of apoptotic cells ( $\mathrm{Wu}$ et al. 2005), the intracytoplasmic signaling pathway for phagocytosis of apoptotic cells mediated by Mer in general remains to be elucidated.

In summary, we demonstrate in the present study that the members of the Tyro 3 RTK subfamily regulate the phagocytic ability of Sertoli cells, and Gas6 can be their functional ligand. Mer may initiate signaling pathways for phagocytosis of apoptotic cells, and Tyro 3 and Axl may simply facilitate Sertoli cells to tether apoptotic spermatogenic cells. The results could provide new insight into the mechanism of phagocytic removal of apoptotic spermatogenic cells by Sertoli cells.

\section{Materials and Methods}

\section{Animals}

The mice singly mutant for each member of the Tyro 3 RTK subfamily were kindly provided by Dr Lemke (Salk Institute for Biological Studies, La Jolla) and maintained in a pathogen-free, temperature- and humidity-controlled room on a $12 \mathrm{~h}$ light: $12 \mathrm{~h}$ darkness cycle. Double and triple knockouts for the members of the Tyro 3 RTK subfamily were produced by crossmating of the single-mutant mice. The WT control mice were the littermates of the mutant mice. The animals had free access to food and water. All the measures taken for the mice were in accordance with approved guidelines (Guideline for the Care and Use of Laboratory Animals) established by the Chinese Council on Animal Care. Three-week-old male mice were used in this study.

\section{Isolation and culture of Sertoli cells}

The procedure for the isolation of Sertoli cells was based on a previous description by Cheng et al. (1986) with some modifications. Briefly, three mice for each occasion were anesthetized with $\mathrm{CO}_{2}$ and killed by cervical dislocation. Decapsulated testes were incubated with $0.5 \mathrm{mg} / \mathrm{ml}$ collagenase (Sigma) at room temperature for $15 \mathrm{~min}$ with gentle oscillation, and then filtered through $80 \mu \mathrm{m}$ copper meshes to eliminate interstitial cells. Seminiferous tubules were resuspended in the collagenase at room temperature for $20 \mathrm{~min}$ to remove myoid cells. The tubules were then incubated with $1 \mathrm{mg} / \mathrm{ml}$ hyaluronidase (Sigma) for $5 \mathrm{~min}$ with gentle oscillation and pipetting. The cells were washed thrice with F12/Dulbecco's modified Eagle medium (DMEM; Gibco), and plated on culture dishes in F12/DMEM supplemented with sodium bicarbonate $(1.2 \mathrm{mg} / \mathrm{ml})$, penicillin $(100 \mathrm{U} / \mathrm{ml})$, streptomycin $(100 \mu \mathrm{g} / \mathrm{ml})$, and $10 \%$ FCS (Gibco). The cells were maintained in a humidified atmosphere of $95 \%$ air: $5 \% \mathrm{CO}_{2}$ (v:v) at $32{ }^{\circ} \mathrm{C}$ for $48 \mathrm{~h}$, and followed by treatment with a hypotonic solution $(20 \mathrm{mM}$ Tris, $\mathrm{pH}$ 7.4) for $2 \mathrm{~min}$ to remove the spermatogenic cells adhering to the Sertoli cells. Twenty-four hours later, the Sertoli cells were detached with trypsin and seeded in 24-well tissue culture plate at $5 \times 10^{4}$ cells/ well and cultured for an additional $24 \mathrm{~h}$ for phagocytosis assays.

\section{Characterizations of primary Sertoli cells}

The primary Sertoli cells used in this study were characterized by examining purity, survival, and metabolic activity. At $24 \mathrm{~h}$ after removing spermatogenic cells by treatment with a hypotonic solution ( $20 \mathrm{mM}$ Tris, pH 7.4), the Sertoli cells were detached and re-seeded for identification of the cells. The purity of primary Sertoli cells was determined by immunofluorescence staining of Wilm's tumor nuclear protein 1 (WT1, a marker of Sertoli cells). Briefly, the Sertoli cells cultured on Lab-Tek chamber slides (Nunc, Naperville, IL, USA) were fixed with cold methanol at $20{ }^{\circ} \mathrm{C}$ for $2 \mathrm{~min}$. The cells were treated with $0.3 \% \mathrm{H}_{2} \mathrm{O}_{2}$ for 15 min and then with $0.3 \%$ Triton X-100 in PBS for another $15 \mathrm{~min}$ at room temperature to increase cell permeability. After blocking by pre-incubation with $10 \%(\mathrm{w} / \mathrm{v})$ normal goat serum in PBS at room temperature for $30 \mathrm{~min}$, the polyclonal anti-WT1 antibody (goat anti-mouse IgG; Santa Cruz Biotechnology, Santa Cruz, CA, USA) was applied at a dilution of 1:200 and incubated at $4{ }^{\circ} \mathrm{C}$ in a moist chamber for overnight. After being washed with PBS thrice, the cells were incubated with the tetramethylrhodamine isothiocyanate (TRITC)-coupled secondary antibody (rabbit anti-goat IgG; Zhongshan, Beijing, China) for $30 \mathrm{~min}$. The cells were 
mounted with Canada balsam (Sigma) after three washes with PBS. The slides were then examined on a fluorescence microscope (IX-71, Olympus).

Cell survival rate was assessed by conventional AO/EB staining. The dye mix for AO/EB (Sigma) staining was $100 \mu \mathrm{g} / \mathrm{ml} \mathrm{AO}$ and $100 \mu \mathrm{g} / \mathrm{ml} \mathrm{EB}$ in PBS. Procedures were followed as described previously (Ribble et al. 2005). Briefly, cells were harvested and pelleted by centrifugation at $120 \mathrm{~g}$ for 5 min. After washing with PBS once, the pellets were re-suspended in $25 \mu \mathrm{l} \mathrm{PBS}$. Subsequently, $2 \mu \mathrm{l}$ AO/EB dye mix were added to the suspension. The cells were viewed and counted under a microscope. Tests were done in triplicate, and a total of 100 cells were counted in each test.

The metabolic activity of cultured Sertoli cells was evaluated by MTT assay. This assay is based on measuring the reduction of yellow tetrazolium (MTT) to a purple formazan facilitated by dehydrogenases of metabolically active cells. Briefly, Sertoli cells were cultured in 96-well plates. After removing culture medium, the cells were then incubated in a medium containing yellow MTT $(0.05 \mathrm{mg} / \mathrm{ml})$. After incubation for an additional $4 \mathrm{~h}$, the cells were dissolved in dimethylsulfoxide (DMSO), and solubilized formazan was quantitated spectrophotometrically at $570 \mathrm{~nm}$ using an EL808 Ultra Microplate Reader (Bio-Tek Instruments, Inc., Winooski, VT, USA). Tests were done in triplicate, and the results were expressed as mean optical density ( \pm S.D.).

\section{Preparation of apoptotic spermatogenic cells}

Spermatogenic cells were prepared as previously described by Shiratsuchi et al. (1997). Briefly, at $48 \mathrm{~h}$ after culture of primary Sertoli cells, the spermatogenic cells suspending in medium were collected, and cultured for another 2 days to induce spontaneous apoptosis. The apoptotic cells were washed with D-Hanks solution, and resuspended in F12/DMEM for phagocytosis assays. All apoptotic germ cells for phagocytosis assays in this study were from WT mice.

\section{Phagocytosis assay}

Phagocytosis of apoptotic spermatogenic cells results in the formation of lipid droplets in Sertoli cells. Therefore, lipid droplets in Sertoli cells were detected by ORO staining and used as a criterion to evaluate the phagocytic ability of Sertoli cells. The procedure of phagocytosis assays was based on a previous protocol (Shiratsuchi et al. 1997) with modifications. At $96 \mathrm{~h}$ after isolation, the Sertoli cells were detached and re-seeded in 24 -well plate at $5 \times 10^{4}$ cells/well. Twenty-four hours later, the cells were washed thrice with D-Hanks solution and cultured in three conditions: F12/DMEM containing $10 \%$ FCS; serum-free medium; and serum-free medium supplemented with Gas6 (R\&D Systems, Inc., Minneapolis, MN, USA). The apoptotic spermatogenic cells were added to the Sertoli cells in 24 -well plates at $5 \times 10^{5}$ cells/well. At $24 \mathrm{~h}$ after co-culture, the apoptotic cells were removed and the Sertoli cells were stained with ORO. Each assay was repeated at least thrice.

Uptake of yellow fluorescent-labeled latex beads of $3 \mu \mathrm{m}$ (Polysciences Inc., Warrington, PA, USA) was used as a measure of the general phagocytic activity of Sertoli cells. The procedure was based on a previous description by Grandjean et al. (1997). Briefly, Sertoli cells were seeded in a 24-well plate at $5 \times 10^{4}$ cells/well. The following day, fluorescent latex beads ( $10^{6}$ in $100 \mu$ l of culture medium) were added to the cultures. At $6 \mathrm{~h}$ after co-culture, cells were washed with D-Hanks solution, and detached by incubating in $1 \mathrm{ml}$ D-Hanks solution containing $0.05 \%$ trypsin for $10 \mathrm{~min}$ at $32{ }^{\circ} \mathrm{C}$. The cells were collected by low-speed centrifugation, and washed twice with D-Hanks solution. The whole procedure efficiently eliminates particles bound to the cells. Inhibition of actin involvement by $50 \mu \mathrm{g}$ cytochalasin B (Sigma) in the phagocytic activity was used as controls. The cells were counted under a fluorescence microscope (IX-71, Olympus). The ratio of the cells having internalized fluorescent beads represented the phagocytic capacity of Sertoli cells. A total of 200 cells were counted at each test, and the results were expressed as the mean value of five tests.

\section{ORO staining}

The co-cultures were washed in PBS for removing suspended apoptotic cells, and the Sertoli cells were fixed with $10 \%$ formalin for $40 \mathrm{~min}$. After a wash with PBS, the cells were stained with ORO (Sigma) solution (ORO saturated solution in isopropanol:water, 3:2) for $15 \mathrm{~min}$ as previously described by Wang et al. (2006). The Sertoli cells were then washed with $70 \%$ alcohol for $5 \mathrm{~s}$ to remove background staining. Finally, the Sertoli cells were rinsed in tap water, counterstained with Harris hematoxylin for $10 \mathrm{~s}$, and mounted in glycerol-PBS (9:1) for observation.

\section{Binding of spermatogenic cells to Sertoli cells}

The binding assay of spermatogenic cells to Sertoli cells was performed as the procedure for the phagocytosis assay except for the following changes: (1) apoptotic spermatogenic cells were fluorescein labeled; (2) cells were washed gently five times with $1 \mathrm{ml}$ PBS after co-culture; and (3) binding rate was scored at $1 \mathrm{~h}$ of incubation of co-cultures. Sertoli cells were counted under a light microscope (IX-71, Olympus) and spermatogenic cells bound to the Sertoli cells were scored with u.v. light. A total of 150 Sertoli cells from three repeat wells were randomly counted for each assay. The assays were done in triplicate.

\section{Morphometric procedures}

The area of lipid droplets in Sertoli cells was quantified using a microscope image analyzer (Image-Pro Express 5.1, Olympus). The area of Sertoli cell nuclei was used as a reference to that of lipid droplets. The area ratio (\%) of lipid droplets to nuclei was used to evaluate phagocytotic ability of Sertoli cells. The morphometric data were analyzed statistically with ANOVA or Student's $t$-test. A total of 200 Sertoli cells from five repeat wells were analyzed for each occasion and the mean values were presented in the results. Each assay was repeated at least thrice. 


\section{RT-PCR}

Total RNA was extracted from Sertoli cells using TRIzol reagent (Gibco) according to the manufacturer's instructions, followed by treatment with RNase-free DNase I (Sigma) at $37{ }^{\circ} \mathrm{C}$ for 20 min to remove potential contaminating genomic DNA. First-strand cDNA was synthesized from the total RNA using MMLV reverse transcriptase (Promega) at $42{ }^{\circ} \mathrm{C}$ for $1 \mathrm{~h}$. PCRs were performed with first-strand CDNA under conditions of $94{ }^{\circ} \mathrm{C}$ for $30 \mathrm{~s}, 54{ }^{\circ} \mathrm{C}$ for $30 \mathrm{~s}$, and $72{ }^{\circ} \mathrm{C}$ for $45 \mathrm{~s}$ for each cycle. Appropriate cycles for different genes were performed to assure that the amplifications were under a linear range. The S16 rRNA gene was used as the control of equal amounts of cDNA in the PCRs. The PCR products were subjected to electrophoresis in $1 \%$ agarose gels, and the bands were analyzed using YLN2000 Gel Analysis System (Yalien, Beijing, China). The PCR was performed using primer pairs as follows: Tyro 3 (419 bp), forward 5'-TGA AGC CCG CAA CAT AAA-3' and reverse 5'-TCC CAT TCC AGG ATA AGG-3'; Axl (398 bp), forward 5'-AGG CTC ATT GGC GTC TGT T-3' and reverse 5'-ATC GCT CTT GCT GGT GTA G-3'; Mer (325 bp), forward $5^{\prime}$-CCT GGA TAT TAG ATG GAC GAA-3' and reverse $5^{\prime}$-AGC AGC CGA GGA TGA TGA A-3 ${ }^{\prime}$; S16 (326 bp), forward 5'-TGT GCT CGG AGC TAT GC- $3^{\prime}$ and reverse $5^{\prime}$-TTC TTG GAG GCT TCA TC-3'.

\section{Acknowledgements}

We sincerely thank Dr Greg Lemke for providing mutant mice. This work was supported by the National Basic Research Program of China (Grant No. 2006CB504001, 2007CB947504) and National Natural Science Foundation of China (Grant No. 30470878, 30570678). The authors declare that there is no conflict of interest that would prejudice the impartiality of this scientific work.

\section{References}

Anderson HA, Maylock CA, Williams JA, Paweletz CP, Shu H \& Shacter E 2003 Serum-derived protein S binds to phosphatidylserine and stimulates the phagocytosis of apoptotic cells. Nature Immunology 4 87-91.

Caraux A, Lu Q, Fernandez N, Riou S, Di Santo JP, Raulet DH, Lemke G \& Roth C 2006 Natural killer cell differentiation driven by Tyro3 receptor tyrosine kinases. Nature Immunology 7 747-754.

Chemes H 1986 The phagocytic function of Sertoli cells: a morphological, biochemical, and endocrinological study of lysosomes and acid phosphatase localization in the rat testis. Endocrinology 119 1673-1681.

Cheng CY, Mather JP, Byer AL \& Bardin CW 1986 Identification of hormonally responsive proteins in primary Sertoli cell culture medium by anionexchange high performance liquid chromatography. Endocrinology 118 480-488.

Cohen PL, Caricchio R, Abraham V, Camenisch TD, Jennette JC, Roubey RA, Earp HS, Matsushima G \& Reap EA 2002 Delayed apoptotic cell clearance and lupus-like autoimmunity in mice lacking the c-mer membrane tyrosine kinase. Journal of Experimental Medicine 196 135-140.

Dym M 1994 Spermatogonial stem cells of the testis. PNAS 91 11287-11289.
Grandjean V, Sage J, Ranc F, Cuzin F \& Rassoulzadegan M 1997 Stagespecific signals in germ line differentiation: control of Sertoli cell phagocytic activity by spermatogenic cells. Developmental Biology $\mathbf{1 8 4}$ 165-174.

Hafizi S \& Dahlback B 2006a Gas6 and protein S. FEBS Journal 273 5231-5244.

Hafizi S \& Dahlback B 2006b Signalling and functional diversity within the Axl subfamily of receptor tyrosine kinases. Cytokine \& Growth Factor Reviews 17 295-304.

Hall MO, Obin MS, Heeb MJ, Burgess BL \& Abrams TA 2005 Both protein S and Gas6 stimulate outer segment phagocytosis by cultured rat retinal pigment epithelial cells. Experimental Eye Research 81 581-591.

Huckins C 1978 The morphology and kinetics of spermatogonial degeneration in normal adult rats: an analysis using a simplified classification of the germinal epithelium. Anatomical Record 190 905-926.

Jegou B 1991 Spermatids are regulators of Sertoli cell function. Annals of the New York Academy of Sciences 637 340-353.

Johnson L, Petty CS \& Neaves WB 1983 Further quantification of human spermatogenesis: germ cell loss during postprophase of meiosis and its relationship to daily sperm production. Biology of Reproduction 29 207-215.

Kerr JB \& de Kretser DM 1974 Proceedings: The role of the Sertoli cell in phagocytosis of the residual bodies of spermatids. Journal of Reproduction and Fertility 36 439-440.

Lemke G \& Lu Q 2003 Macrophage regulation by Tyro 3 family receptors. Current Opinion in Immunology 15 31-36.

Lu Q \& Lemke G 2001 Homeostatic regulation of the immune system by receptor tyrosine kinases of the Tyro 3 family. Science 293 306-311.

Lu Q, Gore M, Zhang Q, Camenisch T, Boast S, Casagranda F, Lai C, Skinner MK, Klein R, Matsushima GK et al. 1999 Tyro-3 family receptors are essential regulators of mammalian spermatogenesis. Nature $\mathbf{3 9 8}$ 723-728.

Manning G, Whyte DB, Martinez R, Hunter T \& Sudarsanam S 2002 The protein kinase complement of the human genome. Science 298 1912-1934.

Miething A 1992 Germ-cell death during prespermatogenesis in the testis of the golden hamster. Cell and Tissue Research 267 583-590.

Nakagawa A, Shiratsuchi A, Tsuda K \& Nakanishi Y 2005 In vivo analysis of phagocytosis of apoptotic cells by testicular Sertoli cells. Molecular Reproduction and Development 71 166-177.

Nakano T, Ishimoto Y, Kishino J, Umeda M, Inoue K, Nagata K, Ohashi K, Mizuno K \& Arita H 1997 Cell adhesion to phosphatidylserine mediated by a product of growth arrest-specific gene 6 . Journal of Biological Chemistry 272 29411-29414.

Oakberg EF 1956 A description of spermiogenesis in the mouse and its use in analysis of the cycle of the seminiferous epithelium and germ cell renewal. American Journal of Anatomy 99 391-413.

Pineau C, Le Magueresse B, Courtens JL \& Jegou B 1991 Study in vitro of the phagocytic function of Sertoli cells in the rat. Cell and Tissue Research 264 589-598.

Prasad D, Rothlin CV, Burrola P, Burstyn-Cohen T, Lu Q, Garcia de Frutos P \& Lemke G 2006 TAM receptor function in the retinal pigment epithelium. Molecular and Cellular Neurosciences 33 96-108.

Ren Y \& Savill J 1998 Apoptosis: the importance of being eaten. Cell Death and Differentiation 5 563-568.

Ribble D, Goldstein NB, Norris DA \& Shellman YG 2005 A simple technique for quantifying apoptosis in 96-well plates. BMC Biotechnology $\mathbf{5} 12$.

Robinson DR, Wu YM \& Lin SF 2000 The protein tyrosine kinase family of the human genome. Oncogene 19 5548-5557.

Russell LD \& Clermont Y 1977 Degeneration of germ cells in normal, hypophysectomized and hormone treated hypophysectomized rats. Anatomical Record 187 347-366.

Schlessinger J 2000 Cell signaling by receptor tyrosine kinases. Cell 103 211-225.

Scott RS, McMahon EJ, Pop SM, Reap EA, Caricchio R, Cohen PL, Earp HS \& Matsushima GK 2001 Phagocytosis and clearance of apoptotic cells is mediated by MER. Nature 411 207-211. 
Shiratsuchi A, Umeda M, Ohba Y \& Nakanishi Y 1997 Recognition of phosphatidylserine on the surface of apoptotic spermatogenic cells and subsequent phagocytosis by Sertoli cells of the rat. Journal of Biological Chemistry 272 2354-2358.

Shiratsuchi A, Kawasaki Y, Ikemoto M, Arai H \& Nakanishi Y 1999 Role of class B scavenger receptor type I in phagocytosis of apoptotic rat spermatogenic cells by Sertoli cells. Journal of Biological Chemistry 274 5901-5908.

Wang H, Chen Y, Ge Y, Ma P, Ma Q, Ma J, Wang H, Xue S \& Han D 2005 Immunoexpression of Tyro 3 family receptors - Tyro 3, Axl, and Mer and their ligand Gas6 in postnatal developing mouse testis. Journal of Histochemistry and Cytochemistry 53 1355-1364.

Wang H, Wang H, Xiong W, Chen Y, Ma Q, Ma J, Ge Y \& Han D 2006 Evaluation on the phagocytosis of apoptotic spermatogenic cells by Sertoli cells in vitro through detecting lipid droplet formation by Oil Red O staining. Reproduction 132 485-492.
Wang H, Chen S, Chen Y, Wang H, Wu H, Tang H, Xiong W, Ma J, Ge Y, Lu Q et al. 2007 The role of Tyro 3 subfamily receptors in the regulation of hemostasis and megakaryocytopoiesis. Haematologica 92 643-650.

Webb JH, Blom AM \& Dahlback B 2003 The binding of protein S and the protein S-C4BP complex to neutrophils is apoptosis dependent. Blood Coagulation \& Fibrinolysis 14 355-359.

Wu Y, Singh S, Georgescu MM \& Birge RB 2005 A role for Mer tyrosine kinase in alphavbeta5 integrin-mediated phagocytosis of apoptotic cells. Journal of Cell Science 118 539-553.

Received 19 June 2007

First decision 2 August 2007

Accepted 24 September 2007 